\title{
Measurement of the Aharonov-Casher geometric phase with a separated-arm atom interferometer
}

\author{
J. Gillot, S. Lepoutre, A. Gauguet, J. Vigué and M. Büchner \\ Laboratoire Collisions Agrégats Réactivité-IRSAMC \\ Université de Toulouse-UPS and CNRS UMR 5589, Toulouse, France \\ PACS 03.75.Dg - Atom and neutron interferometry \\ PACS 03.65.Vf - Phases: geometric; dynamic or topological \\ PACS 39.20.+q - Atom interferometry techniques
}

\begin{abstract}
In this letter, we report a measurement of the Aharonov-Casher (AC) geometric phase with our lithium atom interferometer. The AC phase appears when a particle carrying a magnetic dipole propagates in a transverse electric field. The first measurement of the AC phase was done with a neutron interferometer in 1989 by A. Cimmino et al. (Phys. Rev. Lett. 63, 380, 1989) and all the following experiments were done with Ramsey or Ramsey-Bordé interferometers with molecules or atoms. In our experiment, we use lithium atoms pumped in a single hyperfineZeeman sublevel and we measure the AC-phase by applying opposite electric fields on the two interferometer arms. Our measurements are in good agreement with the expected theoretical values and they prove that this phase is independent of the atom velocity.
\end{abstract}

In 1984, Y. Aharonov, and A. Casher [1] discovered the geometric phase which is called by their name. This phase appears when a particle with a magnetic dipole interacts with an electric field perpendicular to both the particle velocity and to the magnetic dipole. This phase had also been discussed by J. Anandan [2], who did not remark its unusual properties. The Aharonov-Casher (AC) p phase is the second example of a geometric phase, after the Aharonov-Bohm phase 3. A third geometric phase predicted in 1993/1994 by X.-G. He, B.H.J. McKellar 4 and by M. Wilkens 5 is now named the He-McKellar$>$ Wilkens (HMW) phase, and we have recently measured this phase [6, 7]. This phase appears, if an electric dipole travels in a magnetic field $\mathbf{B}$ and if the scalar triple product of the dipole, $\mathbf{B}$ and the velocity vectors is not zero. All these phases belong to the general class of geometric phases discussed by M.V. Berry in 1984 [8,9] and they are very interesting because they strongly differ from dynamical phases: geometric phases modify the wave propagation in the absence of any force on the particle; they are independent of the modulus of the particle velocity but they change sign if the velocity is reversed.

In the present letter, we describe measurements of the AC phase shift using a separated-arm ${ }^{7} \mathrm{Li}$ atom interferometer using Bragg diffraction on laser standing waves.

The internal quantum state of the atom is the same in the two interferometer arms and we apply opposite electric fields and a common magnetic field on the two interferometer arms. The atom fringes are phase shifted by both the $\mathrm{AC}$ and the HMW phases. The AC phase is proportional to the atom magnetic dipole moment, thus depends on the magnetic sublevel, while the HMW phase is independent. By combining measurements made with the ${ }^{7} \mathrm{Li}$ atoms pumped in $F=2$ either in $m_{F}=+2$ or in $m_{F}=-2$, we can extract both phases. In the following, we focus on the AC phase measurements. As explained below, this is the first AC phase measurement of this type and the sensitivity of our atom interferometer has enabled us to test the velocity dependence of this phase.

Since its theoretical discovery, the AC phase has been tested in five different experiments [10-15]. We recall in table 1 the main parameters of these experiments. In the first experiment done in 1989 by A. Cimmino et al. [10] with a neutron interferometer, supplementary phase shifts of magnetic and gravitational origins were needed in order to measure a spin-dependent phase with unpolarized neutrons. The four following experiments were based on Ramsey or Ramsey-Bordé interferometry with atoms or molecules. In these experiments, the atom or molecule propagates in a quantum superposition of internal states with different magnetic moments and the AC phase shift appears directly as a shift of the fringe signal. We do not 
Table 1: Previous measurements of the AC phase. $\mu$ is the magnetic dipole moment and $\mu_{B}$ the Bohr magneton. $E_{m a x}$ is the maximum electric field strength, $\varphi_{A C, \max }$ is the maximum AC phase and $v$ is the particle velocity. Ref. [14] and ref. 15] did not probe the same quantum superposition configuration, which explains the larger ratio $\varphi_{A C, \max } / E_{\max }$ of [15].

\begin{tabular}{|lccccc|}
\hline Species & $\mu / \mu_{B}$ & $\begin{array}{c}E_{\max } \\
(\mathrm{MV} / \mathrm{m})\end{array}$ & $\begin{array}{c}\varphi_{A C, \max } \\
(\mathrm{mrad})\end{array}$ & $\begin{array}{c}\text { error } \\
(\%)\end{array}$ & $\begin{array}{c}v \\
(\mathrm{~m} / \mathrm{s})\end{array}$ \\
\hline $\mathrm{n}$ [10] & $1.0 \times 10^{-3}$ & 30 & 2.19 & 24 & 2680 \\
$\mathrm{TlF}[\mathbf{1 1}]$ & $1.4 \times 10^{-3}$ & 3 & 2.22 & 4 & $220-340$ \\
$\mathrm{TlF}[\mathbf{1 2}]$ & $1.4 \times 10^{-3}$ & 2 & 2.42 & 2 & $188-366$ \\
${ }^{85} \mathrm{Rb}[\mathbf{1 3}$ & $1 / 3$ & 0.9 & 150 & 1.4 & $300-650$ \\
${ }^{40} \mathrm{Ca}[14]$ & $3 / 2$ & 1 & 35 & 2.2 & $643-698$ \\
${ }^{40} \mathrm{Ca}[15]$ & $3 / 2$ & 4.4 & 314 & 2.9 & 650,810 \\
\hline
\end{tabular}

use such quantum superpositions in our experiment: the atom is in the same internal quantum state in the two interferometer arms and we deduce the AC phase by comparing the fringe phase shift measured during experiments made with different atom quantum states.

The AC phase is given by:

$$
\varphi_{A C}=-\frac{1}{\hbar c^{2}} \oint[\mathbf{E}(\mathbf{r}) \times \boldsymbol{\mu}] \cdot d \mathbf{r}
$$

where $\boldsymbol{\mu}$ is the particle magnetic dipole and $\mathbf{E}$ the electric field. A.G. Klein [16] remarked that, at first order in $v / c$, the AC phase can be interpreted as the interaction of the magnetic moment $\boldsymbol{\mu}$ with the motional magnetic field $\mathbf{B}_{\text {mot }} \approx-(\mathbf{v} \times \mathbf{E}) / c^{2}$ seen by the particle in its rest frame moving with the velocity $\mathbf{v}$. In the presence of a magnetic field $\mathbf{B}$, the particle interacts with the total field $\mathbf{B}+\mathbf{B}_{m o t}$ and this interaction induces a phase shift $\varphi_{Z+A C}$ due to the sum of the Zeeman and AC phase effects:

$$
\varphi_{Z+A C}\left(F, m_{F}\right)=-\frac{1}{\hbar} \oint E_{F, m_{F}}\left(\left|\mathbf{B}+\mathbf{B}_{m o t}\right|\right) d t
$$

where $E_{F, m_{F}}\left(\left|\mathbf{B}+\mathbf{B}_{m o t}\right|\right)$ is the energy of the $\left(F, m_{F}\right)$ sublevel in the presence of the field. We measure the AC phase by measuring the variation of $\varphi_{Z+A C}$ due to the presence of $\mathbf{B}_{m o t}$. In our experiment, $B_{m o t} \leq 10^{-8} \mathrm{~T}$ is always considerably smaller than $B \geq 10^{-5} \mathrm{~T}$, and the $\mathrm{AC}$ phase can be written as:

$$
\varphi_{A C}\left(F, m_{F}\right)=-\frac{1}{\hbar} \oint \frac{\partial E_{F, m_{F}}}{\partial B}\left(\mathbf{B}_{m o t} \cdot \hat{\mathbf{e}}_{B}\right) d t
$$

where $\hat{\mathbf{e}}_{B}$ is a unit vector parallel to $\mathbf{B}, \hat{\mathbf{e}}_{B}=\mathbf{B} / B$ : only the component of $\mathbf{B}_{\text {mot }}$ parallel to $\mathbf{B}$ contributes to the AC phase.

Our Mach-Zehnder atom interferometer has been described in detail [17] and it is schematically represented in figure 1. The atomic beam is produced by a supersonic expansion of natural lithium seeded in a large excess of a noble gas which fixes the mean beam velocity $v_{m}$ of the lithium atoms: $v_{m}$ scales like $1 / \sqrt{M}$, where $M$ is the noble gas atomic mass (see table 2). The beam velocity

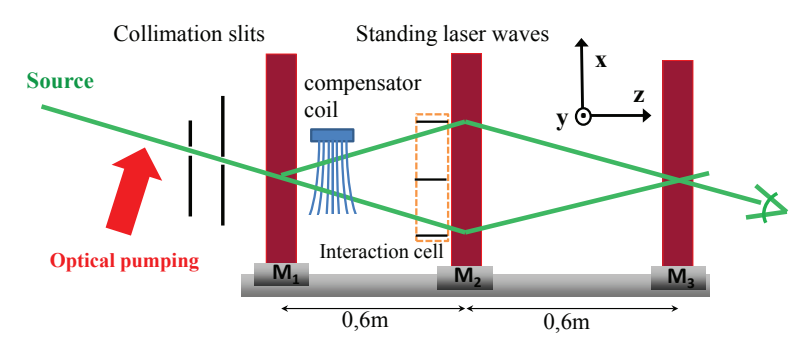

Fig. 1: (Color online) Schematic top-view of our atom interferometer (not to scale): a supersonic lithium is optically pumped, collimated and crosses three laser standing waves, which diffract the atom waves in the Bragg regime. A coil, mounted at mid-distance between the first standing waves produces a compensating magnetic field gradient. The interaction cell is mounted just before the second laser standing wave (see Figure 2 for details.)

distribution is well described by a Gaussian [18, 19] with a $1 / e$ half-width equal to $v_{m} / S_{\|}$where $S_{\|}$is the parallel speed ratio. $S_{\|}$depends on the source parameters (nozzle diameter, pressure, temperature, carrier gas), with typical values $S_{\|}=6-8$ in our experiment.

The lithium beam is optically pumped and collimated. Then, it crosses three laser standing waves which diffract the atom wave in the Bragg regime: first-order diffraction is used to split, reflect and recombine the atomic waves. The laser used to produce the standing waves is a single frequency dye laser. Its wavelength $\lambda_{L}$ is chosen on the blue side of the ${ }^{2} \mathrm{~S}_{1 / 2} \rightarrow{ }^{2} \mathrm{P}_{3 / 2}$ transition of ${ }^{7} \mathrm{Li}$ at 671 $\mathrm{nm}$ : this choice and the natural abundance of ${ }^{7} \mathrm{Li}(92.5 \%)$ explain the fact, that only ${ }^{7} \mathrm{Li}$ contributes to the interferometer signal [17,20]. This signal can be written as:

$$
I=I_{0}\left[1+\mathcal{V} \cos \left(\varphi_{d}+\varphi_{p}\right)\right]
$$

where $I_{0}$ is the mean signal intensity and $\mathcal{V}$ the fringe visibility. $\varphi_{d}$ is the diffraction phase, $\varphi_{d}=4 \pi\left(x_{1}-2 x_{2}+\right.$ $\left.x_{3}\right) / \lambda_{L}$ with $x_{i}$ the position of the mirror $M_{i}(\mathrm{i}=1,2,3)$, and $\varphi_{p}$ the phase due to various perturbations. The phase sensitivity, i.e. the minimum detectable phase for an one-second data recording is proportional to $\varphi_{\min }=$ 


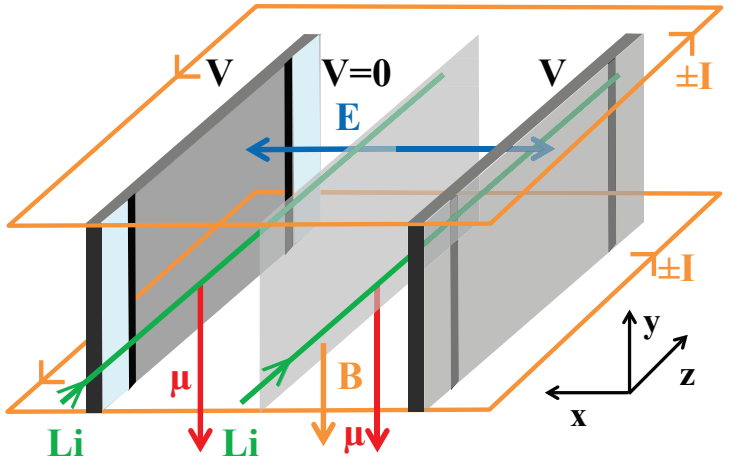

Fig. 2: (Color online) Schematic drawing of our interaction cell (not to scale). A double capacitor produces opposite electric fields $\mathbf{E}$ (blue) in the horizontal plane. The high voltage electrodes are surrounded by grounded guard electrodes (light blue). Two coils (yellow) produce a vertical magnetic field $\mathbf{B}$. The magnetic dipole (red) $\boldsymbol{\mu}$ is parallel or antiparallel to the magnetic field, depending on the pumped level. Each interferometers arm (green) passes through a different capacity.

$1 /\left(\mathcal{V} \sqrt{I_{0}}\right)$. Table 2 summarizes the typical values of $I_{0}$, $\mathcal{V}$ and $\varphi_{\min }$ as a function of the carrier gas used in our experiment.

Table 2: The mean lithium beam velocity $v_{m}$, the mean signal intensity $I_{0}$, the fringe visibility $\mathcal{V}$ and the phase sensitivity $\varphi_{\min }$ measured with the three carrier gases. $I_{0}$ is large with neon and argon but considerably smaller with krypton, while $\mathcal{V}$ has opposite variations. $\varphi_{\min }$ has comparable values with neon and argon and is about half as much with krypton.

\begin{tabular}{|c|c|c|c|c|}
\hline $\begin{array}{l}\text { carrier gas } \\
M \text { (a.m.u.) }\end{array}$ & $\begin{array}{c}v_{m} \\
(\mathrm{~m} / \mathrm{s})\end{array}$ & $\begin{array}{c}I_{0} \\
\left(10^{3} \mathrm{c} / \mathrm{s}\right)\end{array}$ & $\begin{array}{l}\mathcal{V} \\
\%\end{array}$ & $\begin{array}{c}\varphi_{\min } \\
\operatorname{mrad} / \sqrt{\mathrm{Hz} z}\end{array}$ \\
\hline Ne: 20.2 & $1520 \pm 38$ & 56 & 60 & 7.0 \\
\hline Ar: 39.9 & $1062 \pm 20$ & 33 & 75 & 7.3 \\
\hline Kr: 83.8 & $744 \pm 18$ & 7 & 80 & 14.9 \\
\hline
\end{tabular}

The lithium de Broglie wavelength is $\lambda_{d B}=5.7 \times$ $10^{-8} / v_{m}$, with $\lambda_{d B}$ in $\mathrm{m}$ and $v_{m}$ in $\mathrm{m} / \mathrm{s}$, and the first order diffraction angle is $\theta=2 \lambda_{d B} / \lambda_{L} \approx 0.17 / v_{m} \mathrm{rad}$, for example, with argon as a carrier gas, $\lambda_{d B} \approx 54 \mathrm{pm}$ and $\theta \approx 160 \mu \mathrm{rad}$. The distance between the interferometer arms, which is maximum at the second laser standing wave, is approximately equal to 143 (krypton), 100 (argon) and $70 \mu \mathrm{m}$ (neon), which is sufficient to introduce a septum 21 between the two interferometer arms.

We have built the present experiment to measure the HMW phase shift, using an arrangement inspired by the ideas of $\mathrm{H}$. Wei et al. 22, with opposite electric fields on the two interferometer arms and a common homogeneous magnetic field 6, 23. Figure 2 shows the interaction cell. It consists of a double plane capacitor used to produce the needed electric fields and the capacitor assembly is inserted in a support which holds two coils producing the magnetic field.

The two capacitors share the septum as a common, grounded electrode. The electric field vector lies on the horizontal plane, with opposite values on the two interferometer arms and a magnitude of $E_{\max } \approx 0.7 \mathrm{MV} / \mathrm{m}$ for the maximum applied voltage $V=800 \mathrm{~V}$ and it extends over a $48 \mathrm{~mm}$ length. Each high voltage electrode is separated by $1 \mathrm{~mm}$-wide gaps from two $5 \mathrm{~mm}$-long grounded guard electrodes, in order to have well defined fringing fields.

The two coils produce a magnetic field $\mathbf{B}$ along the vertical axis and with a current of $I=40 \mathrm{~A}$, we obtain a field strength of $B_{\text {coil }}=22.4 \mathrm{mT}$. This magnetic field is needed for the observation of the HMW effect but its presence is also important for the observation of the $\mathrm{AC}$ phase, as discussed in equation (3) and also because the magnetic moment of a hyperfine-Zeeman sublevel is parallel or antiparallel to the local magnetic field. The magnetic field produced in the interaction region has a small gradient along the $x$-direction so that it has slightly different values on the two interferometer arms, which results in a Zeeman phase shift. We have introduced a compensator coil to produce an opposite gradient, thus canceling the Zeeman phase shift. This compensator coil is located at mid-distance between the first and second laser standing waves (see figure 10.

As already mentioned, we optically pump the lithium atoms in one Zeeman-hyperfine ground state sublevel $F=$ $2, m_{F}=+2$ (or $\left.m_{F}=-2\right)$ 24]. The optical pumping is performed before beam collimation in order to avoid heating the transverse motion by exchange of photon momenta (radiation pressure). We control the magnetic field in the pumping region by three pairs of square Helmholtz coils. The D1 line of lithium is used because of the larger hyperfine splitting of the ${ }^{2} P_{1 / 2}$ state. Two circularly polarized laser beams are tuned to the ${ }^{2} S_{1 / 2}, F=1 \rightarrow{ }^{2} P_{1 / 2}, F=2$ and the ${ }^{2} S_{1 / 2}, F=2 \rightarrow{ }^{2} P_{1 / 2}, F=2$ transitions. The first laser beam empties the $F=1$ level, while the second one pumps the atoms into the $F=2, m_{F}= \pm 2$ level, depending on the chosen circular polarization and on the magnetic field direction.

As discussed below, we separate the HMW and AC phases by reversing the $m_{F}$ value which is obtained by reversing the magnetic field in the pumping region. The $m_{F}$ value measured on an axis parallel to the local magnetic field is conserved along the atom propagation, if the condition for an adiabatic transport is fulfilled and our measurements of the pumping efficiency rule out any Majorana spin-flip of the optically pumped atoms along their propagation. We have characterized the pumping efficiency by an atom interferometric method and we have found that the pumped sublevel has a fraction of the total population near $95 \pm 5 \%$ 24. Our results are recalled in table 3 .

For the ${ }^{2} S_{1 / 2}, F=2, m_{F}= \pm 2$ sublevels of ${ }^{7} \mathrm{Li}$, $\left|\mu / \mu_{B}\right| \approx 1$. Using eq. (3) and assuming $\mathbf{B}_{m o t}$ parallel to $\mathbf{e}_{B}$, we predict an $\mathrm{AC}$ phase slope $\left|\partial \varphi_{A C} / \partial V\right|=$ $(8.57 \pm 0.05) \times 10^{-5} \mathrm{rad} / \mathrm{V}$ with an uncertainty due to the 
Table 3: The measured population $P\left(F=2, m_{F}\right)$ after optical pumping for different atom velocities $v_{m}$ [24].

\begin{tabular}{l|r|r}
$v_{m}(\mathrm{~m} / \mathrm{s})$ & $m_{F}$ & $P\left(F=2, m_{F}\right)$ \\
\hline 744 & +2 & $(96 \pm 6) \%$ \\
& -2 & $(93 \pm 7) \%$ \\
1062 & +2 & $(100 \pm 13) \%$ \\
& -2 & $(95 \pm 11) \%$ \\
1520 & +2 & $(90 \pm 1) \%$ \\
& -2 & $(94 \pm 2) \%$
\end{tabular}

capacitor geometry. We thus predict $\left|\varphi_{A C}\right| \approx 69 \mathrm{mrad}$ for the largest voltage $V=800 \mathrm{~V}$.

As explained in our papers [6, 7, 23, we eliminate interferometer phase drifts by alternating 6 field configurations, $(V, I),(V, 0),(0, I),(-V, 0),(-V, I)$ and $(0,0)$ during each 20 second-long fringe scan. Least-square fits are used to extract the phase $\varphi\left(V, I, m_{F}\right)$ corresponding to each field configuration and to an optical pumping in the $F=2, m_{F}=+2$ sublevel. We reduce the statistical uncertainty by averaging the results of about 80 similar fringe scans. Successive experiments are made with opposite values of the current $I$ and of the $m_{F}$ value. Five effects contribute to the measured phase shifts. There are two related to the residual polarizability and Zeeman phases due to the differences of electric fields and magnetic fields on the two interferometer arms. The third concerns the HMW phase $\varphi_{H M W}(V, I)$ and two others the AC phases $\varphi_{A C}(V, I)$ and $\varphi_{A C}(V, I=0)$. The following combination cancels the residual Stark and Zeeman phases:

$$
\varphi_{E B}\left(V, I, m_{F}\right)=\varphi(V, I)-\varphi(V, 0)-\varphi(0, I)+\varphi(0,0)
$$

and $\varphi_{E B}\left(V, I, m_{F}\right)$ is equal to:

$$
\begin{aligned}
\varphi_{E B}\left(V, I, m_{F}\right)= & \varphi_{H M W}(V, I)+\varphi_{A C}\left(V, I, m_{F}\right) \\
& -\varphi_{A C}\left(V, I=0, m_{F}\right)
\end{aligned}
$$

Following equation (3), $\varphi_{A C}\left(V, I=0, m_{F}\right)$ is sensitive to the laboratory residual magnetic field $\mathbf{B}_{l a b}$ when $I=0$. $B_{l a b}$ is about $35 \mu \mathrm{T}$ and the field is mainly oriented downwards along the $y$-axis. If we apply a coil current $I \geq 5 \mathrm{~A}$, the coil magnetic field $B_{\text {coil }}$ is always larger than $2.8 \mathrm{mT}$ and $\mathbf{B}=\mathbf{B}_{\text {coil }}+\mathbf{B}_{l a b}$ is nearly perfectly vertical. As the HMW phase does not depend on $m_{F}$ while the AC phase changes sign with $m_{F}$, we extract the contributions due to the $\mathrm{AC}$ phase thanks to the linear combination :

$$
\varphi_{A C}^{\exp }(V, I)=\left[\varphi_{E B}(V, I, 2)-\varphi_{E B}(V, I,-2)\right] / 2
$$

Following equation (6),$\varphi_{A C}^{\exp }(V, I)$ should be equal to the theoretical value:

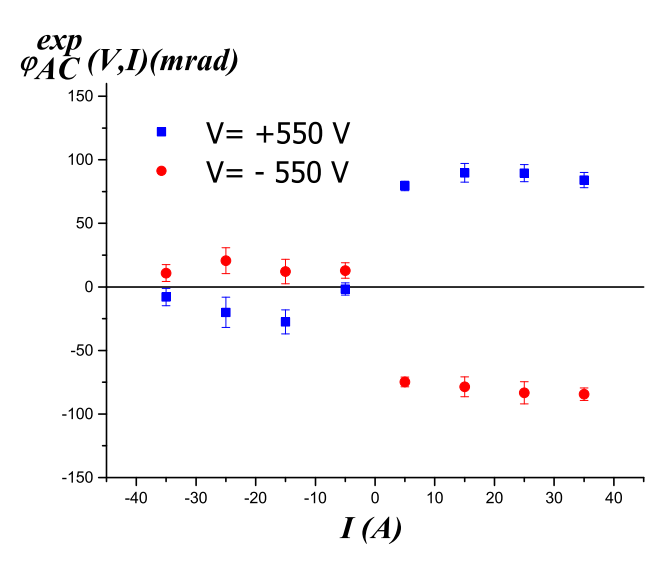

Fig. 3: (Color online) The AC phase $\varphi_{A C}^{e x p}(V, I)$ defined by equation (7) is plotted as a function of the applied current for two voltages $V= \pm 550 \mathrm{~V}$. The sign $\varphi_{A C}\left(V, I, m_{F}\right)$ is related to the sign of $I$ and its absolute value is larger than $\varphi_{A C}(V, I=$ $\left.0, m_{F}\right)$, due to the better verticality of $\mathbf{B}$, as shown by equation (3).

$$
\varphi_{A C}^{\exp }(V, I)=\varphi_{A C}(V, I, 2)-\varphi_{A C}(V, I=0,2)
$$

Figure 3 shows typical measurements of the AC phase $\varphi_{A C}^{\exp }(V, I)$ for two voltages $V= \pm 550 \mathrm{~V}$ as a function of the current $I$. For a given sense of $I, \varphi_{A C}^{\exp }(V, I)$ does not vary significantly with the current as expected but $\varphi_{A C}^{e x p}(V, I)$ changes sign when we reverse the current $I$ and the magnetic field $\mathbf{B}$. For $I \leq-5 \mathrm{~A}, \mathrm{~B}$ is obviously pointing in a direction opposite to the one of $\mathbf{B}_{l a b}$ and $\varphi_{A C}\left(V, I, m_{F}\right)$ has a sign opposite to the one of $\varphi_{A C}\left(V, I=0, m_{F}\right)$. As the modulus of $\varphi_{A C}\left(V, I, m_{F}\right)$ is larger than the one of $\varphi_{A C}\left(V, I=0, m_{F}\right)$, the modulus of $\varphi_{A C}^{\exp }(V, I)$ is small but non vanishing when $I \leq-5 \mathrm{~A}$. For $I \geq 5 \mathrm{~A}$, the situation is reversed, with $\varphi_{A C}\left(V, I, m_{F}\right)$ and $\varphi_{A C}\left(V, I=0, m_{F}\right)$ having the same sign and adding their contributions to $\varphi_{A C}^{\exp }(V, I)$.

Using equation (8), the AC phase in the presence of the applied magnetic field $\mathbf{B}, \varphi_{A C}(V, I, 2)$, can be extracted by the linear combination:

$$
\varphi_{A C}(V,|I|)=\frac{1}{2}\left[\varphi_{A C}^{\exp }(V, I)-\varphi_{A C}^{\exp }(V,-I)\right]
$$

and $\varphi_{A C}(V,|I|)$ must be equal to $\varphi_{A C}(V, I, 2)$. Figure 4 shows $\varphi_{A C}(V,|I|)$ as a function of $V$ for different values for $|I|$.

The data points plotted with their $1 \sigma$ error bars represent a quasi linear behaviour and a least-square fit provides a slope equal to $(-8.51 \pm 0.18) \times 10^{-5} \mathrm{rad} / \mathrm{V}$ and a zero-compatible offset $(0.2 \pm 1.2) \mathrm{mrad}$. We performed similar experiments and data analysis with the three different mean velocities of the lithium beam. The results are collected in Table 4 and plotted in Figure 5.

However, the optical pumping is not perfect and we must take this into account for a more realistic estimate 


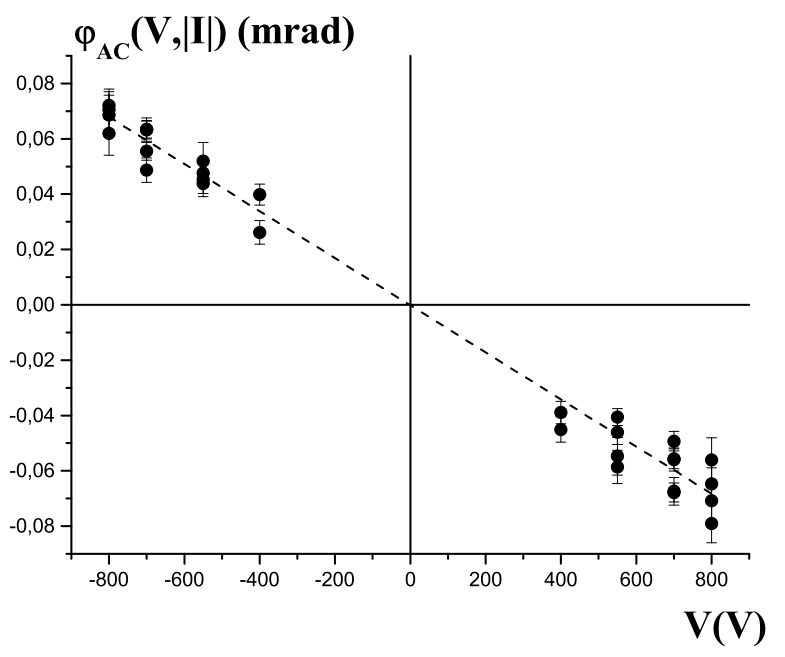

Fig. 4: The AC phase $\varphi_{A C}(V,|I|)$, defined by equation (9), is plotted as a function of the applied voltage $V$ for an experiment with a mean lithium velocity $v_{m}=1062 \mathrm{~m} / \mathrm{s}$. The data points (black circles) exhibit a linear behaviour and the dashed line represents the best least-square fit.

of the AC phase. The interferometer signal (eq. (4)) is the sum of the contributions of the 8 Zeeman-hyperfine sublevels:

$$
\begin{aligned}
I= & I_{0} \sum_{F, m_{F}} P\left(F, m_{F}\right) \\
& {\left[1+\mathcal{V}\left(F, m_{F}\right) \cos \left(\varphi_{d}+\varphi_{A C}\left(F, m_{F}\right)\right)\right] }
\end{aligned}
$$

where $P\left(F, m_{F}\right)$ is the normalized population of the $F, m_{F}$ sublevel and, for simplicity, we have omitted all the perturbation phases except the AC phase. The contributions of the $m_{F} \neq \pm 2$ sublevels play a minor role for three reasons: their populations are small, the AC phase change sign with the sublevel and the visibility $\mathcal{V}\left(F, m_{F}\right)$ of their contributions should be small, because the compensator produces a low magnetic field which compensates exactly the Zeeman phase shift only for the $m_{F}= \pm 2$ sublevels for which the Zeeman effect is purely linear. As a consequence, we consider that the contributions from the $m_{F} \neq \pm 2$ sublevels can be neglected and then, a straightforward calculation shows that our experiment measures a corrected AC phase with a weight function of the populations of the populations $P\left(F=2, m_{F}= \pm 2\right)$ :

$$
\varphi_{A C}^{c}\left(m_{F}\right)=\varphi_{A C}\left(m_{F}\right) \frac{P\left(2, m_{F}\right)-P\left(2,-m_{F}\right)}{P\left(2, m_{F}\right)+P\left(2,-m_{F}\right)}
$$

when the optical pumping aims at populating the $F=$ $2, m_{F}$ sublevel. We thus get more realistic theoretical estimates of the measured AC phases (see Table 4) with errors bars which take into account the errors on the population measurements 24] and on the capacitor geometry.
Table 4: Our measurements of the AC phase slope $\partial \varphi_{A C} / \partial V$ in $10^{-5} \mathrm{rad} / \mathrm{V}$ are compared to the theoretical values corrected for imperfect optical pumping (see eq. (10)).

\begin{tabular}{c|c|c}
$v_{m}(\mathrm{~m} / \mathrm{s})$ & $\begin{array}{c}\partial \varphi_{A C} / \partial V \\
\text { experiment }\end{array}$ & $\begin{array}{c}\partial \varphi_{A C}^{c} / \partial V \\
\text { theory }\end{array}$ \\
\hline $1520 \pm 18$ & $-8.05 \pm 0.20$ & $-7.86 \pm 0.12$ \\
$1062 \pm 20$ & $-8.51 \pm 0.18$ & $-8.51 \pm 0.88$ \\
$744 \pm 18$ & $-8.44 \pm 0.41$ & $-8.40 \pm 0.48$
\end{tabular}

Our measurements are in excellent agreement with these corrected theoretical values. Moreover, our results are in very good agreement with an $\mathrm{AC}$ phase independent of the atom velocity, as illustrated in Figure 5 .

In conclusion, we have measured the Aharanov-Casher (AC) phase by atom interferometry using optically pumped lithium atoms. Our separated arm interferometer operates with atoms in a single internal quantum state and two opposite electric fields are applied on the interferometer arms to realize the AC measurements. This approach to measure the $\mathrm{AC}$ phase is new, as all previous works used either unpolarized neutrons [10] or Ramsey or Ramsey-Bordé interferometers with atoms and molecules 11 15 .

Our measurements were performed during the study of the He-McKellar-Wilkens (HMW) phase and we had not optimized the setup for the measurement of the AC phase. In particular, a better control of the residual magnetic field in the interaction region would have simplified the analysis. We have verified that the AC phase depends linearly on the electric field strength. We have performed AC phase measurements for three different mean velocities of the atomic beam (744, 1062 and $1520 \mathrm{~m} / \mathrm{s}$ ) and these measurements are in good agreement with the fact that this phase does not depend on the atom velocity, a characteristic of a geometric phase. Our measurements, with a statistical uncertainty close to $3 \%$, agree with their theoretical estimates, which have a considerably larger uncertainty due to their sensitivity to the population distribution over the Zeeman-hyperfine sublevels. The optical pumping of the lithium beam is quite efficient, with about $95 \pm 5 \%$ of the population transferred in the pumped sublevel, but a better pumping, which is feasible [27], would reduce the uncertainty on the theoretical values of the $\mathrm{AC}$ phase.

$$
* * *
$$

We thank the laboratory technical and administrative staff for their help. We thank H. Batelaan and A. Cronin for fruitful discussions, G. Trénec, A. Miffre and M. Jacquey for all the work done on our atom interferometer. We thank CNRS INP, ANR (grants ANR05-BLAN-0094 and ANR-11-BS04-016-01 HIPATI) and Région Midi-Pyrénées for support. 


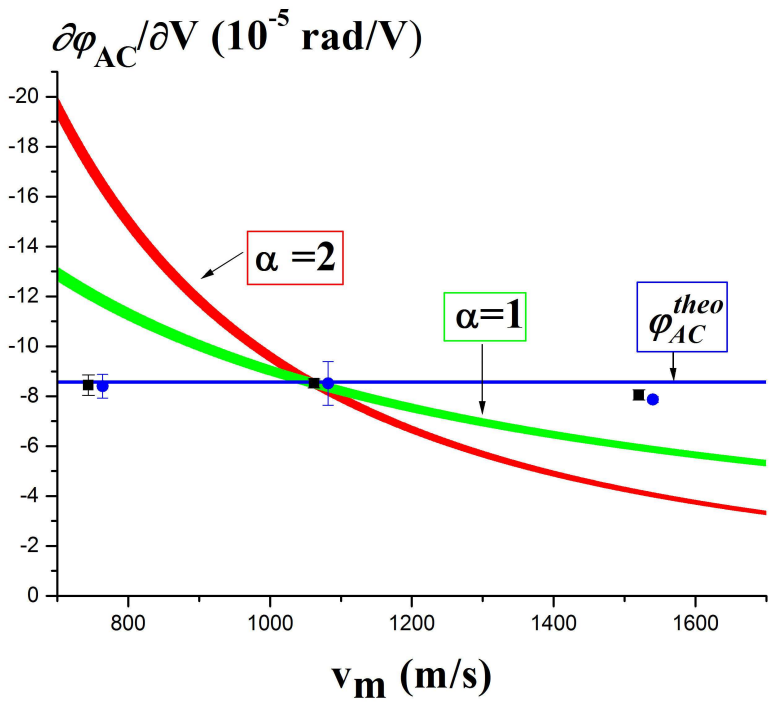

Fig. 5: (Color online) Plot of the slope of the AC phase $\partial \varphi_{A C}(V) / \partial V$ as a function of the mean atom velocity $v_{m}$. The experimental results (black squares) are compared to the theoretical value (blue horizontal band) which assumes a perfect optical pumping. The corrected theoretical values are represented by blue circles slightly displaced at the right of the experimental values for better visibility). The shaded areas represent what would be the phase if, starting from its value for $v_{m}=1062 \mathrm{~m} / \mathrm{s}$, the AC phase was varying like $1 / v_{m}^{\alpha}$ with $\alpha=1$ (green) or $\alpha=2$ (red).

\section{REFERENCES}

[1] Aharonov Y. and Casher A., Phys. Rev. Lett., 53 (1984) 319

[2] Anandan J., Phys. Rev. Lett., 48 (1982) 1660

[3] Aharonov Y. and Bohm A., Phys. Rev., 115 (1959) 485

[4] He X.-G. and McKellar B. H. J., Phys. Rev. A, 47 (1993) 3424

[5] Wilkens M., Phys. Rev. Lett., 72 (1994) 5

[6] Lepoutre S., Gauguet A., Trénec G., Büchner M. and Vigué J., Phys. Rev. Lett., 109 (2012) 120404

[7] Gillot J., Lepoutre S., Gauguet A., Büchner M. and Vigué J., Phys. Rev. Lett., 111 (2013) 030401

[8] Berry M.V., Proc. R. Soc. Lond., A392 (1984) 45

[9] Shapere A. and WilczeK F., Geometric Phases in Physics (World Scientific ed., Singapore) 1989

[10] Cimmino A., Opat G. I., Klein A. G., Kaiser H., Werner S. A., Arif M. and Clothier R., Phys. Rev. Lett., 63 (1989) 380

[11] Sangster K., Hinds E. A., Barnett S. M. and RiIs E., Phys. Rev. Lett., 71 (1993) 3641

[12] Sangster K., Hinds E. A., Barnett S. M. and SinClair A. G., Phys. Rev. A, 51 (1995) 1776

[13] Görlitz A., Schun B. and Weis A., Phys. Rev. A, 51 (1995) R4305

[14] Zeiske K., Zinner G., Riehle F. and Helmcke J., Appl. Phys. B, 60 (1995) 205

[15] Yanagimachi S., Kajiro M., Machiya M. and MoriNaga A., Phys. Rev. A, 65 (2002) 042104
[16] Klein A. G., Physica, 137B (1986) 230

[17] Miffre A., Jacquey M., Büchner M., Trénec G. and Vigué J., Eur. Phys. J. D, 33 (2005) 99

[18] Toennies J. P. and Winkelmann K., J. Chem. Phys., 66 (1977) 3965

[19] Miller D. R., Atomic and molecular beam methods, edited by Scoles G. (Oxford University Press) 1998, pp. 14-53

[20] Jacquey M., Miffre A., BüChner M., Trénec G. and Vigué J., Europhys. Lett. 77 (2007) 20007

[21] Ekstrom C. R., Schmiedmayer J., Chapman M. S., Hammond T. D. and Pritchard D. E., Phys. Rev. A, 51 (1995) 3883

[22] Wei H., Han R. and Wei X., Phys. Rev. Lett., 75 (1995) 2071

[23] Lepoutre S., Gillot J., Gauguet A., Büchner M. and Vigué J., Phys. Rev. A, 88 (2013) 043628

[24] Gillot J., Gauguet A., Büchner M. and Vigué J., Optical pumping of a lithium atomic beam for atom interferometry, to be published in Eur. Phys. J. D (accepted 30 oct. 2013, preprint: arxiv.org/abs/1305.6727)

[25] Miffre A., Jacquey M., Büchner M., Trénec G. and Vigué J., Eur. Phys. J. D, 38 (2006) 353

[26] Cronin A. D., Schmiedmayer J. and Pritchard D. E., Rev. Mod. Phys., 81 (2009) 1051

[27] Schinn G. W., Han X. L. and Gallagher A., J. Opt. Soc. Am. B, 8 (1991) 169 